\title{
Analysis of Natural Frequency Sensitivity of Truck Body
}

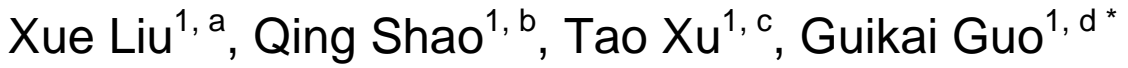 \\ ${ }^{1}$ School of Mechanical Science and Engineering, Jilin University, PR China \\ a181231735@qq.com, bshaoqing14@mails.jlu.edu.cn, xutao@jlu.edu.cn, dggk@jlu.edu.cn
}

Keywords: Sensitivity analysis, Vibration reanalysis, Natural frequency sensitivity, Frequency shift combined approximations.

\begin{abstract}
Sensitivity is extremely important in computer aided material and structural design. The present paper focuses on the sensitivity with respect to the design variables of truck body. This paper proposes a solution procedure of truck body for natural frequency sensitivity calculation in vibration analysis and optimization using a frequency shift combined approximations (FSCA) approach. These sensitivity analysis formulations are based on the reduced basis method and finite difference derivative. Using a shift coefficient, accurate sensitivities of natural frequency can be obtained. Truck body with large degrees of freedom demonstrates the accuracy of the sensitivity reanalysis method. Compared to the exact solution, the proposed method is efficient and easy to implement. With the sensitivity information, satisfying materials and thicknesses can be selected. The proposed method is suitable for material and structural design and optimization.
\end{abstract}

\section{Introduction}

Design sensitivity analysis of materials and structures deals with the calculation of changes of the response caused by the parameters of the structure. The derivatives of responses with respect to the parameters, called the sensitivity information, are commonly used in various problems of engineering analysis and optimization, such as selecting search direction and generating approximations for response of modification.

Several approximate or exact reanalysis methods were established to analyze structures which are changed in process of design and optimization [1]. Reanalysis of structure for linear static problems have been developed since the 1970s [2]. Combined Approximations (CA) approach is one of the most effective methods for solving static displacement equations [3]. After CA method was founded, some extended CA methods were proposed [4].

Vibration reanalysis methods have been discussed since the early 2000s [5]. Kirsch grafted the CA approach to solve eigenproblems [6], where approximation results can be obtained by solving a smaller eigenproblem in the reduced subspace composed of several approximation vectors. Combining CA and Rayleigh quotient, Chen developed an extended CA method of eigenproblem reanalysis for large modifications [7]. A modified combined approximations(MCA) method for reanalysis of dynamic problems with many dominant mode shapes was discussed by Geng Zhang [8]. With a suitable frequency-shift, the CA approach allowed to calculate higher modes accurately [9].

In the optimization procedures of the design process, the sensitivity information is usually needed. But the low computational efficiency due to the repetitive computations is a main obstacle. Therefore, based on CA, sensitivity of displacement was present by Kirsch [10]. Recently, analytical sensitivity reanalysis of static displacement was investigated by using Taylor series expansion and CA method [11]. Reanalysis of eigenvalue and eigenvector sensitivities with CA method when the eigenvalues are repeated was considered by Zhao [12].

In this study, a novel sensitivity reanalysis of vibration problem is proposed to acquire the derivatives of the eigenvalues, where a new set of basis vectors is calculated by the FSCA method which is compared with traditional CA method. Then approximate sensitivities solution for the original eigenproblem is achieved. The formulas of sensitivity reanalysis are expressed, and a 
numerical example of truck body is demonstrated for the accuracy. Finally, conclusion is summarized.

\section{Methods}

Reanalysis Method for Eigenproblems. Analysis of natural frequency sensitivity is aimed to find which parameter is most important for the natural frequency response of structure within the whole changing parameters, which plays an important role in structural optimization. The solution of large structural sensitivity analysis is one of the most costly computations in the structural design cycle.

In practice, natural frequency has the same mean with eigenvalue in the mathematical model of structural analysis problems. In many applications, it is common for only the eigenvalues which are smallest in modulus to be required.

For a structure with stiffness and mass matrices $\underset{n \times n}{\mathbf{K}^{(0)}}$ and $\underset{n \times n}{\mathbf{\mathbf { M } ^ { ( 0 ) }}}$ respectively, the equation of the first $\mathrm{m}$ eigenproblems for structure is

$$
\begin{aligned}
& \underset{n \times n}{\mathbf{K}_{n \times m}^{(0)}} \underset{n \times m}{\boldsymbol{\Phi}^{(0)}}=\underset{n \times n}{\mathbf{M}^{(0)}} \underset{n \times m}{\boldsymbol{\Phi}^{(0)}} \underset{m \times m}{\mathbf{\Lambda}^{(0)}} \\
& \underset{n \times n}{\mathbf{K}_{n \times n}^{(0)}}=\underset{n \times n}{\mathbf{U}_{0}^{T}} \mathbf{U}_{n \times n}
\end{aligned}
$$

where, $\underset{m \times m}{\Lambda_{(0)}}$ and $\underset{n \times m}{\boldsymbol{\Phi}^{(0)}}$ denote the matrix of eigenvalues for initial structure and the corresponding matrix of eigenvectors, $n$ is the number of degrees of the structural freedom, $\mathbf{U}_{n \times n}$ in Eq. 2 is an upper triangular matrix obtained with Cholesky decomposition of $\mathbf{K}_{n \times n}^{(0)}$.

When there are changes in the stiffness and mass matrices, $\underset{n \times n}{\mathbf{K}}=\underset{n \times n}{\mathbf{K}(0)}+\underset{n \times n}{\Delta \mathbf{K}}$ and $\underset{n \times n}{\mathbf{M}}=\underset{n \times n}{\mathbf{M}^{(0)}}+\underset{n \times n}{\Delta \mathbf{M}}$, respectively, the eigenproblem of the modified structure is expressed as follows:

$$
\underset{n \times n}{\mathbf{K}} \underset{n \times m}{\boldsymbol{\Phi}}=\underset{n \times n}{\mathbf{M}} \underset{n \times m}{\boldsymbol{\Phi}} \underset{m \times m}{\boldsymbol{\Lambda}}
$$

where, $\underset{m \times m}{\Lambda}$ and $\underset{n \times m}{\boldsymbol{\Phi}}$ represent the matrix of eigenvalues and the matrix of corresponding eigenvectors for the modified structure, respectively.

CA Method. The matrix of eigenvectors $\underset{n \times m}{\mathbf{\Phi}}$ can be approximated by a linear combination of preselected $s$ basis vectors as follows:

$$
\underset{n \times s}{\mathbf{R}}=\left[\underset{n \times 1}{\mathbf{r}^{(1)}}, \mathbf{r}_{n \times 1}^{(2)}, \cdots, \mathbf{r}_{n \times 1}^{(s)}\right]
$$

where, $s$ is the number of basis vectors $\underset{n \times 1}{\mathbf{r}^{(i)}}, i=1,2, \cdots, s$, and $\underset{n \times s}{\mathbf{R}}$ is the matrix formed by basis vectors.

The basis vectors are given by Eq. 5 and Eq. 6 .

$$
\begin{aligned}
& \mathbf{r}_{n \times 1}^{(i+1)}=-\mathbf{K}_{n \times n}^{(0)-1} \underset{n \times n}{\Delta \mathbf{K} \mathbf{r}_{n \times 1}^{(i)}}, i=1,2, \cdots, s \\
& \underset{n \times 1}{\mathbf{r}^{(1)}}=\mathbf{K}_{n \times n}^{(0)-1} \underset{n \times n}{\mathbf{M}} \boldsymbol{\varphi}_{n \times 1}^{(0)}{ }_{n \times 1}, k=1,2, \cdots, m
\end{aligned}
$$

Using Eq. 2, Cholesky decomposition of $\mathbf{K}_{n \times n}^{(0)}$ can be obtained with the original analysis, calculation of $\mathbf{r}_{n \times 1}^{(i+1)}$ involve only forward and backward substitutions. We first solve for $\underset{n \times 1}{\mathbf{z}}$ by the forward substitution

$$
\underset{n \times n}{\mathbf{U}_{0}^{T}} \mathbf{z}=-\underset{n \times n}{\Delta \mathbf{K}} \mathbf{r}_{n \times 1}^{(i)}, i=1,2, \cdots, s
$$

Then $\mathbf{r}_{n \times 1}^{(i+1)}$ is calculated by the backward substitution

$$
\underset{n \times n}{\mathbf{U}_{0}} \mathbf{r}_{n \times 1}^{(i+1)}=\underset{n \times 1}{\mathbf{Z}}, i=1,2, \cdots, s
$$


Similarly, the $\underset{n \times 1}{\mathbf{r}^{(1)}}$ is calculated from

$$
\underset{n \times n}{\mathbf{U}_{0}^{T}} \mathbf{U}_{n \times n} \mathbf{r}_{n \times 1}^{(1)}=\underset{n \times n}{\mathbf{M}} \boldsymbol{\varphi}_{n \times 1}^{(0)}{ }_{k}, k=1,2, \cdots, m
$$

The stiffness matrix $\underset{n \times n}{\mathbf{K}}$ and mass matrix $\underset{n \times n}{\mathbf{M}}$ are condensed as Eq. 10, respectively.

$$
\underset{s \times s}{\mathbf{K}_{R}}=\underset{n \times s}{\mathbf{R}} \underset{n \times n \times n \times s}{\mathbf{K}} \mathbf{R} \underset{s \times s}{\mathbf{M}}=\underset{n \times s}{\mathbf{R}} \mathbf{R}_{n \times n \times n \times s}^{T} \mathbf{M}
$$

where, $\mathbf{K}_{R}$ and $\mathbf{M}_{R}$ denote condensed stiffness matrix and mass matrix, respectively.

The eigenproblem equation are condensed to a smaller Eq. 11 with an unknown $\underset{s \times 1}{\mathbf{y}_{1}}$. The $k$ th eigenvector $\boldsymbol{\varphi}_{n \times 1}$ is calculated by Eq. 12 .

$$
\begin{aligned}
& \underset{s \times s}{\mathbf{K}_{k} \mathbf{y}_{1}}=\lambda_{1} \mathbf{M}_{s \times 1} \mathbf{M}_{s \times s} \mathbf{y}_{1 \times 1} \\
& \underset{n \times 1}{\boldsymbol{\varphi}_{k}}=y_{1} \mathbf{r}_{n \times 1}+y_{2} \underset{n \times 1}{\mathbf{r}_{2}+\cdots+y_{s}} \mathbf{r}_{n \times 1}=\underset{n \times s_{s \times 1}}{\mathbf{R}} \mathbf{y}_{s \times 1}
\end{aligned}
$$

FSCA Method. To obtain eigenvectors $\underset{n \times m}{\boldsymbol{\Phi}}$, postmultiplying Eq. 3 by $\underset{m \times m}{\mathbf{A}^{-1}}$, then Eq. 13 is given.

$$
\underset{n \times n \times m \times m}{\mathbf{K}} \underset{m \times m}{\boldsymbol{\Lambda}} \boldsymbol{\Lambda}^{-1}=\underset{n \times n \times n \times m}{\mathbf{M}} \boldsymbol{\Phi}
$$

Subtract Eq. 13 on both sides with $\mu^{-1} \mathbf{K} \mathbf{\Phi}$ and rearrange

$$
\underset{n \times m}{\mathbf{\Phi}}=\left(\underset{n \times n}{\mathbf{M}}-\mu^{-1} \underset{n \times n}{\mathbf{K}}\right)^{-1} \underset{n \times n}{\mathbf{K}} \underset{n \times m}{\mathbf{\Phi}}\left(\underset{m \times m}{\boldsymbol{\Lambda}^{-1}-\mu^{-1}} \underset{m \times m}{\mathbf{I}}\right)
$$

Precisely, given $\underset{n \times m}{\boldsymbol{\Phi}^{(i)}}$, one can compute $\underset{n \times m}{\boldsymbol{\Phi}^{(i+1)}}$ by solving iterative formula as Eq. 15 .

$$
\underset{n \times m}{\mathbf{\Phi}^{(i+1)}}=\left(\underset{n \times n}{\mathbf{M}-} \mu^{-1} \underset{n \times n}{\mathbf{K}}\right)^{-1} \underset{n \times n}{\mathbf{K}} \underset{n \times m}{\mathbf{\Phi}} \underset{m \times m}{(i)}\left(\boldsymbol{\Lambda}^{-1}-\mu^{-1} \underset{m \times m}{\mathbf{I}}\right)
$$

To accelerate the convergence of $\underset{n \times m}{\boldsymbol{\Phi}^{(i+1)}}$, assuming that a linear combination of $\underset{n \times m}{\boldsymbol{\Phi}^{(i)}}$, where $i=0,1, \cdots, s-1$, is closer to the exact solution than $\underset{n \times m}{\boldsymbol{\Phi}^{(i)}}$ :

$$
\begin{aligned}
& \underset{n \times m}{\boldsymbol{\Phi}_{c}}=a_{0} \underset{n \times m}{\boldsymbol{\Phi}^{(0)}}+a_{1} \underset{n \times m}{\boldsymbol{\Phi}_{(1)}^{(1)}}+a_{2} \underset{n \times m}{\boldsymbol{\Phi}^{(2)}}+\cdots+a_{s-1} \underset{n \times m}{\mathbf{\Phi}_{n \times m}^{(s-1)}}
\end{aligned}
$$

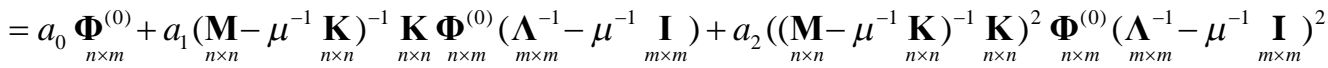

$$
\begin{aligned}
& +\cdots+a_{s-1}\left(\left(\underset{n \times n}{\mathbf{M}-} \mu^{-1} \underset{n \times n}{\mathbf{K}}\right)^{-1} \underset{n \times n}{\mathbf{K}}\right)^{s-1} \underset{n \times m}{\mathbf{\Phi}} \underset{m \times m}{(0)}\left(\mathbf{\Lambda}^{-1}-\mu^{-1} \underset{m \times m}{\mathbf{I}}\right)^{s-1}
\end{aligned}
$$

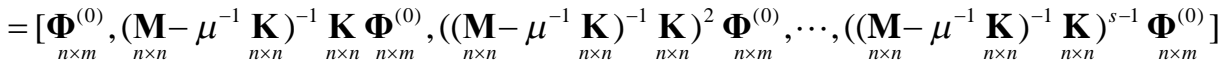

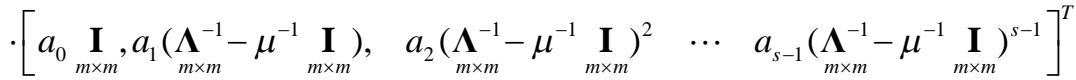

$$
\begin{aligned}
& =\underset{n \times m s}{\mathbf{R}} \underset{m s \times m}{\mathbf{X}}
\end{aligned}
$$

where, $a_{i}, i=1,2, \cdots, s-1$ are defined as undetermined relaxation factors in linear combination. $\underset{n \times m m s}{\mathbf{R}}$ and $\underset{m \times \times m}{\mathbf{X}}$ are given by Eq. 17 and Eq. 18, respectively.

$$
\begin{aligned}
& \underset{n \times m s}{\mathbf{R}}=\left[\underset{n \times m}{\boldsymbol{\Phi}^{(0)}},\left(\mathbf{M}-\mu^{-1} \underset{n \times m}{\mathbf{K})^{-1}} \mathbf{K} \boldsymbol{\Phi}^{(0)},\left(\left(\mathbf{M}-\mu^{-1} \underset{n \times m}{\left.\mathbf{K})^{-1} \mathbf{K}\right)^{2}} \mathbf{\Phi}^{(0)}, \cdots,\left(\left(\mathbf{M}-\mu^{-1} \mathbf{K}\right)_{n \times m}^{-1} \mathbf{K}\right)^{s-1} \mathbf{\Phi}^{(0)}\right]\right.\right.\right. \\
& \underset{m \times \times m}{\mathbf{X}}=\left[\begin{array}{lllll}
a_{0} & \mathbf{I} \times m \\
\mathbf{I} & a_{1}\left(\underset{m \times m}{\left(\boldsymbol{\Lambda}^{-1}-\mu^{-1}\right.} \underset{m \times m}{\mathbf{I}}\right), \quad a_{2}\left(\underset{m \times m}{\boldsymbol{\Lambda}^{-1}-\mu^{-1}} \underset{m \times m}{\mathbf{I}}\right)^{2} \quad \cdots \quad a_{s-1}\left(\underset{m \times m}{\left(\boldsymbol{\Lambda}^{-1}-\mu^{-1}\right.} \underset{m \times m}{\mathbf{I}}\right)^{s-1}
\end{array}\right]^{T}
\end{aligned}
$$

Premultiplying Eq. 3 by $\mathbf{R}_{n \times m s}^{T}$, the condensed eigenproblem can be expressed as follows:

$$
\underset{n \times m s}{\mathbf{R}^{T}} \underset{n \times n}{\mathbf{K}} \underset{n \times m s}{\mathbf{R}} \underset{m \times \times m}{\mathbf{X}}=\mathbf{R}_{n \times m s}^{\mathbf{R}^{T}} \underset{n \times n \times n \times m s}{\mathbf{M}} \underset{n \times \times m m}{\mathbf{X}} \underset{m \times m}{\Lambda}
$$




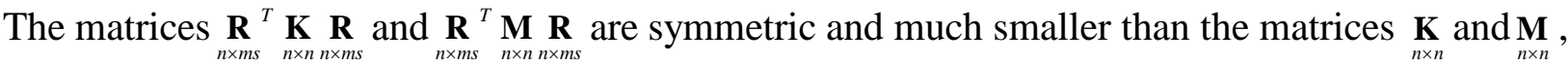
respectively. Rather than computing the exact solution by solving the large $n \times n$ eigenproblem in Eq. 3, we solve the smaller $m s \times m s$ system in Eq. 19 for $\underset{m s \times m}{\mathbf{X}}$ and $\underset{m \times m}{\Lambda}$ instead.

The advantage of the shift factor is that more accuracy results can be obtained. To improve the accuracy of higher modes calculation, the highest mode vector is chosen to generate the frequency shift factor.

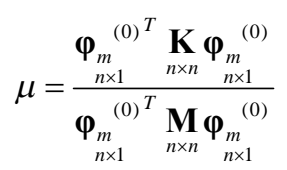

where, $\varphi_{m \times 1}^{\left({ }^{(0)}\right.}, i=0, \cdots, s-1$ is the mode vector with respect to the highest natural frequency of interest.

Exact Sensitivity Analysis of Eigenvalue. Differentiating Eq. 1 with respect to $\theta$ and rearranging gives

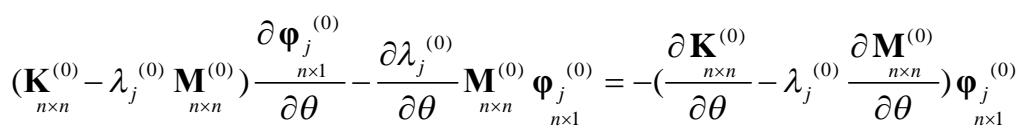

The derivatives of eigenvalues may be obtained by pre-multiplying Eq. 21 by $\boldsymbol{\varphi}_{j}^{(0) T}$ and rearranging.

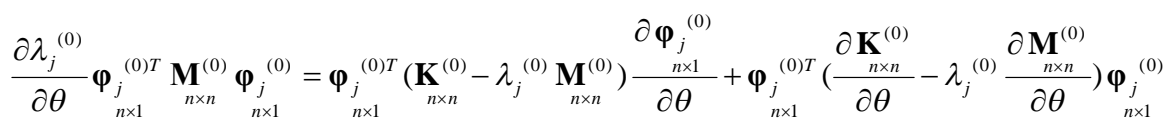

Eq. 1 can be written as $\underset{n \times 1}{\boldsymbol{\varphi}_{j}^{(0) T}}\left(\underset{n \times n}{\mathbf{K}^{(0)}}-\lambda_{j}^{(0)} \underset{n \times n}{\mathbf{M}_{n \times n}^{(0)}}\right)=\underset{1 \times n}{0}$, and when the eigenvectors are mass narmalized

$$
\boldsymbol{\varphi}_{j \times 1}^{(0) T} \underset{n \times n}{\mathbf{M}_{n \times 1}^{(0)}} \boldsymbol{\varphi}_{j \times 1}^{(0)}=1
$$

Noting that mass and stiffness matrices are symmetric, we obtain an expression for the eigenvalue sensitivity:

$$
\frac{\partial \lambda_{j}^{(0)}}{\partial \theta}=\boldsymbol{\varphi}_{j \times 1}^{(0) T}\left(\frac{\partial \mathbf{K}_{n \times n}^{(0)}}{\partial \theta}-\lambda_{j}^{(0)} \frac{\partial \mathbf{M}_{n \times n}^{(0)}}{\partial \theta}\right) \underset{n \times 1}{\left({ }^{(0)}\right.}
$$

Sensitivity Analysis using Finite Difference Derivatives. Consider the problem of calculating the derivatives $\frac{\partial \lambda_{j}^{(0)}}{\partial \theta}$ of the eigenvector $\lambda_{j}$ with respect to a design variable $\theta$ at the point $\theta^{(0)}$. In the central-difference approximation, the derivatives are approximated from the eigenvalue at the perturbed point $\theta^{(0)}+\delta \theta$ and $\theta^{(0)}-\delta \theta$ by

$$
\frac{\partial \lambda_{j}^{(0)}}{\partial \theta}=\frac{\lambda_{j}\left(\theta^{(0)}+\delta \theta\right)-\lambda_{j}\left(\theta^{(0)}-\delta \theta\right)}{2 \delta \theta}
$$

where, $\delta \theta$ is a predetermined step-size. Finite difference methods are easy to implement and therefore they are attractive in many applications. Eq. 25 requires calculation at two points. For a problem with $\mathrm{n}$ design variables, finite difference derivative calculations require repetition of the analysis for $2 \mathrm{n}+1$ different design points. This procedure is usually not efficient by exact eigenvalue analysis. By solving a much smaller eigenproblem using FSCA method, the efficiency can be improved.

\section{Calculations}

To demonstrate the accuracy of the derivatives calculation using FSCA method, large finite element truck body model example is presented. 
The Young's modulus of the material is $E=2.1 \times 10^{11} \mathrm{~Pa}$; the mass density is $\rho=7.8 \times 10^{3} \mathrm{~kg} / \mathrm{m}^{3}$; the Poisson's ratio is 0.3 . The truck body contains 1896 elements, 1944 nodes and 11664 degrees of freedom. The truck body, design variables and original values of the truck body are signed in Fig. 1. Comparisons of the natural frequency sensitivities for the first 3 orders are listed in Table 1.
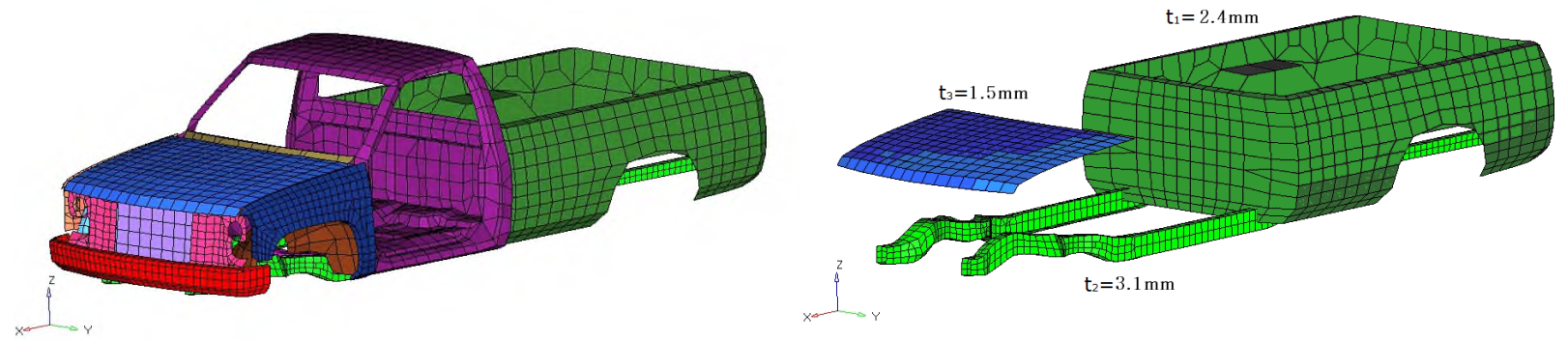

Fig. 1 The truck body, design variables and the original values.

Table 1. Comparisons of the natural frequency sensitivities for the first 3 orders. (Hz/mm)

\begin{tabular}{|c|c|c|c|c|c|c|}
\hline \multirow[t]{2}{*}{ Mode order } & \multirow[t]{2}{*}{$\begin{array}{c}\text { Design } \\
\text { variables }\end{array}$} & \multirow[t]{2}{*}{ Exact } & \multicolumn{2}{|c|}{$\begin{array}{c}\text { CA with } \\
\text { central-difference } \\
\text { approximation }\end{array}$} & \multicolumn{2}{|c|}{$\begin{array}{c}\text { FSCA with } \\
\text { central-difference } \\
\text { approximation }\end{array}$} \\
\hline & & & $\mathrm{s}=3$ & Error $(\%)$ & $\mathrm{s}=3$ & Error $(\%)$ \\
\hline \multirow{3}{*}{$1^{\text {st }}$} & $\mathrm{t}_{1}$ & 2.94953 & 2.93251 & -0.577 & 2.94024 & -0.315 \\
\hline & $\mathrm{t}_{2}$ & 0.00217 & 0.00225 & 3.687 & 0.00216 & -0.461 \\
\hline & $\mathrm{t}_{3}$ & 0.00003 & 0.00004 & 33.333 & 0.00003 & 0.000 \\
\hline \multirow{3}{*}{$2^{\text {nd }}$} & $\mathrm{t}_{1}$ & 3.00946 & 2.99984 & -0.320 & 3.00422 & -0.174 \\
\hline & $\mathrm{t}_{2}$ & 0.01747 & 0.01777 & 1.717 & 0.01740 & -0.401 \\
\hline & $\mathrm{t}_{3}$ & 0.00119 & 0.00114 & -4.202 & 0.00124 & 4.202 \\
\hline \multirow{3}{*}{$3^{\mathrm{rd}}$} & $\mathrm{t}_{1}$ & 0.23546 & 0.23435 & -0.471 & 0.23478 & -0.289 \\
\hline & $\mathrm{t}_{2}$ & 0.16558 & 0.16734 & 1.063 & 0.16587 & 0.175 \\
\hline & $\mathrm{t}_{3}$ & 1.01384 & 1.01465 & 0.080 & 1.01396 & 0.012 \\
\hline
\end{tabular}

\section{Conclusions}

A sensitivity analysis procedure for natural frequency based on FSCA and finite difference derivative is proposed here. A truck body example verifies the accuracy of the present method, which is also compared to the exact solution and the traditional CA method with finite difference derivative simultaneously. According to the results listed in Table 1, with only 3 basis vectors selected, errors from proposed method compared to the exact solution are less than $0.5 \%$ only except the sensitivity for the 2 nd order mode with respect to the design parameter $t_{3}$. The results computed with FSCA method is much better than those calculated by traditional CA method. The matrix order of solution equation can be reduced from $11664 * 11664$ to $30 * 30$ by using FSCA method. And the efficiency is improved obviously. It is expected that the sensitivity analysis using FSCA could reduce the computational cost in problems where repeated analysis is needed in material and structural dynamic optimization. 


\section{Acknowledgments}

This work was supported by the National Science Foundation of China (Grant No. 11502092), and the Plan for Scientific and Technological Development of Jilin Province (Grant Nos. 20140520111JH and 20160520064JH)

\section{References}

[1] U. Kirsch, Reanalysis and sensitivity reanalysis by combined approximations, Struct. Multidiscip. Optim. 40 (2010) 1-15.

[2] S. R. Phansalkar, Matrix iterative methods for structural reanalysis, Comput. Struct. 4(4) (1974) 779-800.

[3] U. Kirsch, Implementation of combined approximations in structural optimization, Comput. Struct. 78(1-3) (2000) 449-457.

[4] W. Zuo, Z. Yu, S. Zhao, W. Zhang, A hybrid Fox and Kirsch's reduced basis method for structural static reanalysis, Struct. Multidiscip. Optim. 46(2) (2012) 261-272.

[5] R. Sun, D. Liu, T. Xu, H. Zhang, W. Zuo, New adaptive technique of Kirsch method for structural reanalysis, AIAA J. 52(3) (2014) 486-495.

[6] U. Kirsch, M. Bogomolni, Nonlinear and dynamic structural analysis using combined approximations, Comput. Struct. 85(10) (2007) 566-578.

[7] U. Kirsch, Approximate vibration reanalysis of structures, AIAA J. 41(3) (2003) 504-511.

[8] S. H. Chen, X. W. Yang, Extended Kirsch combined method for eigenvalue reanalysis, AIAA J. 38(5) (2000) 927-930.

[9] S. H. Chen, X. M. Wu, Z. J. Yang, Eigensolution reanalysis of modified structures using epsilon-algorithm, Int. J. Numer. Meth. Eng. 66(13) (2006) 2115-2130.

[10] G. Zhang, E. Nikolaidis, Z. P. Mourelatos, An efficient re-analysis methodology for probabilistic vibration of large-scale structures, J. Mech. Des. 1(1) (2009) 36-56.

[11] T. Xu, G. Guo, H. Zhang, Vibration reanalysis using frequency-shift combined approximations, Struct. Multidiscip. Optim. 44(2) (2011) 235-246.

[12] U. Kirsch, P. Y. Papalambros, Accurate displacement derivatives for structural optimization using approximate reanalysis, Comput. Method. Appl. M. 190(31) (2001) 3945-3956.

[13] W. Zuo, J. Bai, J. Yu, Sensitivity reanalysis of static displacement using taylor series expansion and combined approximate method, Struct. Multidiscip. Optim. 53(5) (2016) 953-959.

[14]U. Kirsch, M. Bogomolni, Efficient finite difference design sensitivities, AIAA J. 43(2) (2005) 399-405.

[15] S. Zhao, G. Guo, W. Zhang, D. Liu, Efficient procedures of sensitivity analysis for structural vibration systems with repeated frequencies, J. Appl. Math. 2013 (2013) 1-7. 